\title{
Genomic analyses identify signaling pathways and biological processes in Human papillomavirus (HPV) infected oral cancer patients
}

\author{
Fang Wang \\ Tingxiang Chang \\ Hongmei Guo \\ Hanming Gu ( $\square$ laygmp@gmail.com ) \\ James Liu
}

\section{Research Article}

Keywords:

Posted Date: January 25th, 2022

DOI: https://doi.org/10.21203/rs.3.rs-1293225/v1

License: (c) (i) This work is licensed under a Creative Commons Attribution 4.0 International License.

Read Full License 


\section{Abstract}

Human papillomavirus (HPV) is a critical cause of the head and neck squamous cell carcinoma. However, the molecular mechanism and biological function of HPV in oral cancer remains unknown. In this study, our aim is to identify the significant molecules and pathways by analyzing the RNA-seq data. The GSE184616 was produced by the Illumina NovaSeq 6000 (Homo sapiens). The KEGG and GO analyses showed PI3K-Akt signaling pathway and Extracellular matrix are the main processes during oral cancer with HPV infection. Moreover, we identified the top ten key molecules including FN1, ACTB, COL3A1, COL5A1, COL4A1], COL4A2, COL5A2, MMP9, COL6A2, BGN. Therefore, our study may provide new insights into the treatment of oral cancer.

\section{Introduction}

Head and neck squamous cell cancers initiated from the epithelium are the most common malignancies in the head and neck ${ }^{1}$. The rich prevalence of HNSCC is due to the consumption of specific cancerinducing products such as tobacco, alcohol, and areca nut products, and the rising rates of HPV infection have recently resulted in the high prevalence of HNSCC in the US ${ }^{2}$. Men have higher risks than women in the development of oral cancer ${ }^{3}$. The survival rates for oral cancer have improved, for example, the 5-year survival rate has increased to $66 \%$ in the period $2002-2006$ by analyzing across all age groups ${ }^{4}$.

Most oral cancers are linked to the infection with oncogenic strains of human papillomavirus (HPV) including HPV-16 and HPV-18 ${ }^{5}$. HPV-positive oral cancer shows a more favorable prognosis than HPVnegative oral cancer, and recent studies show that lower-dose chemoradiation is effective in HPV-positive diseases $^{6}$. Oral cancer is characterized by genetic instability. The transition from dysplasia to carcinoma is related to the loss of $3 p 21,17 p 13,11 q 13,13 q 21$, and 14q32 in the site of TP532. However, whether the progression of oral cancer is closely dependent on the sequence alteration or accumulation remains unresolved.

In this study, we analyzed the effects of HPV infection on oral cancers by using the RNA-seq data. We identified a variety of DEGs and significant biological processes. We also performed the gene enrichment and the protein-protein interaction (PPI) network to obtain the interacting molecules. The biological genes and pathways in our study will improve the treatment of oral cancer.

\section{Methods}

Data resources

Gene dataset GSE184616 was obtained from the GEO database. The data was produced by the Illumina NovaSeq 6000 (Homo sapiens) (School of Mathematics and Statistics, University of Sydney, City Road, Camperdown, NSW, Australia). The analyzed dataset includes 5 of controls (HPV negative patients) and 5 of HPV positive patients. 
The data were organized and conducted by $\mathrm{R}$ package as previously described ${ }^{7-9}$. We used a classical $\mathrm{t}-$ test to identify DEGs with $\mathrm{P}<0.01$ and fold change $\geq 1.5$ as being statistically significant.

The Kyoto Encyclopedia of Genes and Genomes (KEGG) and Gene Ontology (GO)

KEGG and GO analyses were conducted by the R package (ClusterProfiler) and Reactome. $P<0.05$ was considered statistically significant.

Protein-protein interaction (PPI) networks

The Molecular Complex Detection (MCODE) of Cytoscope was used to create the PPI networks. The significant modules were produced from constructed PPI networks. The pathway analyses were performed by using Reactome (https://reactome.org/), and $\mathrm{P}<0.05$ was considered significant.

\section{Results}

\section{Identification of DEGs in oral cancer between HPV-negative and HPV-positive patients}

To determine the effects of HPV on oral cancer, we analyzed the RNA-seq data from the HPV-negative and -positive oral squamous cell carcinoma Patients. A total of 575 genes were identified with the threshold of $P<0.01$. The top up- and down-regulated genes were indicated by the heatmap and volcano plot (Figure 1). The top ten DEGs were listed in Table 1.

\section{Enrichment analysis of DEGs between HPV-negative and HPV-positive patients}

To further understand the potential biological processes, we performed the KEGG and GO analyses (Figure 2). The significant KEGG items includes "PI3K-Akt signaling pathway", "Human papillomavirus infection", "Focal adhesion", "ECM-receptor interaction", "Small cell lung cancer", "Amoebiasis", and "Arrhythmogenic right ventricular cardiomyopathy". We identified the BP of GO including "Extracellular matrix organization", "Extracellular structure organization”, "External encapsulating structure organization”, "Connective tissue development”, "Collagen metabolic process”, "Collagen fibril organization”, “Endodermal cell differentiation”, “Endoderm formation”, “Basement membrane organization", and "Protein hydroxylation". We identified the CC of GO including "Collagen-containing extracellular matrix", “Endoplasmic reticulum lumen”, “Focal adhesion”, "cell-substrate junction”, "basement membrane", "collagen trimer", "lysosomal lumen", "complex of collagen trimers", "microfibril", and "fibrillar collagen trimer". We then identified the MF of GO including "extracellular matrix structural constituent", "growth factor binding", "collagen binding", "metalloendopeptidase activity", "integrin binding", "iron ion binding", "extracellular matrix structural constituent conferring tensile strength", "extracellular matrix binding", "L-ascorbic acid binding", and "platelet-derived growth factor binding". 


\section{PPI network analysis}

To determine the potential relationship among the DEGs, we constructed the PPI network by using 509 nodes and 1417 edges. We set the combined score $>0.2$ as a cutoff by using the Cytoscope software. Table 2 showed the top ten genes with the highest scores. The top two significant modules were indicated in Figure 3. We further analyzed the PPI and DEGs with Reactome map (Figure 4) and identified the top ten functional processes including "Extracellular matrix organization", "Collagen formation", "Assembly of collagen fibrils and other multimeric structures", "Collagen biosynthesis and modifying enzymes", "ECM proteoglycans", "Non-integrin membrane-ECM interactions", "Post-translational protein phosphorylation", "Degradation of the extracellular matrix", "Elastic fibre formation", and "Laminin interactions" (Supplemental Table S1).

\section{Discussion}

Recent publications showed that HPV does not lead to genital and anal cancer but is a direct cause for the progression of oral cancer ${ }^{10}$. Interestingly, several studies also found that HPV DNA is present in the OSCC, but their interactive relationships are still unclear ${ }^{1}$. Thus, further studies focusing on larger genomic data are needed to decipher risk factors for oral cancers.

The KEGG and GO analyses showed that "PI3K-Akt signaling pathway" and "Extracellular matrix" are the main processes during the oral cancer with HPV infection. Sadhna Aggarwal et al found PI3K inhibitors (PI-828 and PI-103) inhibit the growth and proliferation of oral cancer cells by activating the apoptosis and changing the cell cycles ${ }^{11}$. An-Jie Jhou et al found chlorpromazine promotes the G2/M phase arrest and apoptosis through mediating the PI3K/ATK pathways in human oral cancer ${ }^{12}$. Moreover, they also found chlorpromazine represses tumor growth in zebrafish and mouse oral cancer models ${ }^{12}$. Franziska $\mathrm{E}$ Marquard et al found $90 \%$ of oral cancer showed the activation of PI3K/AKT/mTOR signaling. And the activated $\mathrm{PI} 3 \mathrm{~K} / \mathrm{AK} T$ signaling is associated with radiotherapy and drug resistance ${ }^{13}$. One feature of cancer is the degradation of the extracellular matrix ${ }^{14}$. For example, the matrix metalloproteinases in oral cancers break down the extracellular matrix to allow cancer cells to spread. Moreover, the increased expression of MM9 is a marker of poor prognosis ${ }^{15}$. Elias Sundquist et al also found that the neoplastic extracellular matrix environment boosts cancer in vitro studies ${ }^{16}$.

In addition, we identified the significant interactive molecules by creating the PPI network in oral cancer with HPV infection, which may help to explain the effects of HPV infection. Yoshihiro Morita et al found that fibronectin 1 increases the expression of VEGF-C, lymphangiogenic, and lymph node metastasis in oral cancer ${ }^{17}$. Interestingly, the upregulation of fibronectin 1 results in oral cancer by the activation of FAK, which suggests that fibronectin 1-FAK signaling may be a potential target for oral cancer ${ }^{17}$. ACTB is closely related to a number of cancers, which is disordered in the liver, melanoma, renal, colorectal, gastric, lung, and breast cancers. The dysregulated ACTB causes the malfunction of the cytoskeleton to further promote the invasiveness and metastasis of cancers $^{18}$. Su Young Oh et al found COL3A1 is significantly decreased in the saliva of oral cancer patients, which may be a potential biomarker for the 
prediction of oral cancer ${ }^{19}$. GPCR and RGS proteins play essential roles in different diseases including heart, brain, lung, bone diseases, and a variety of inflammatory diseases ${ }^{20-22}$ and cancers ${ }^{23-31}$. Interestingly, GPR56, a GPCR from the family of adhesion GPCRs, can interact with COL3A1 to inhibit neural migration ${ }^{32}$. Hung-Chih Chen et al found COL5A1 is upregulated in tumor tissues and a high level of COL5A1 is related to the late stage of oral cancer and lymph node metastasis ${ }^{33}$. Moreover, they also found that the high expression level of COL5A2 was associated with better disease-free survival in oral cancer patients $^{33}$. Xiao-Zhi Lü et al found that COL4A1, COL4A2, and COL5A2 mRNAs in the oral cancer tissues are increased in comparison to the normal samples ${ }^{34}$. Li-Sung Hsu et al found KLF6 can inhibit oral cancer migration and invasion by decreasing the MMP9 activities ${ }^{35}$. Stéphane Grauzam found NEDD9 induced MMP9 secretion is invadopodia formation in oral cancer cells ${ }^{36}$. Circadian clocks and their downstream pathways play key roles in cellular functions (proliferation, differentiation, and secretion) and human diseases (fat, metabolic syndrome, arthritis, and aging) ${ }^{37-48}$. Jian Wang found MMP9 is a critical downstream of Bmal1, which is able to promote the invasion and metastasis of cancer $^{49}$. Yang Liu et al found the biglycan is increased in endometrial cancer, which is correlated with histopathological grade and poor prognosis of endometrial cancer ${ }^{50}$.

In summary, our study discovered the significant effects of HPV on oral cancers. The "PI3K-Akt signaling pathway" and "Extracellular matrix" signaling pathways are the major affected processes during the development of oral cancer. Our study provides valuable guidance for the treatment of oral cancer.

\section{Declarations}

\section{Author Contributions}

Fang Wang, Tingxiang Chang, Hongmei Guo: Methodology and Writing. Hanming Gu, James Liu: Conceptualization, Writing- Reviewing and Editing.

\section{Funding}

This work was not supported by any funding.

\section{Declarations of interest}

There is no conflict of interest to declare.

\section{References}

1. Economopoulou P, de Bree R, Kotsantis I, Psyrri A: Diagnostic Tumor Markers in Head and Neck Squamous Cell Carcinoma (HNSCC) in the Clinical Setting. Front Oncol 2019, 9:827.

2. Johnson DE, Burtness B, Leemans CR, Lui VWY, Bauman JE, Grandis JR: Head and neck squamous cell carcinoma. Nat Rev Dis Primers 2020, 6:92. 
3. Suba Z: Gender-related hormonal risk factors for oral cancer. Pathol Oncol Res 2007, 13:195-202.

4. Pulte $\mathrm{D}$, Brenner $\mathrm{H}$ : Changes in survival in head and neck cancers in the late 20th and early $21 \mathrm{st}$ century: a period analysis. Oncologist 2010, 15:994-1001.

5. Burd EM: Human papillomavirus and cervical cancer. Clin Microbiol Rev 2003, 16:1-17.

6. Elrefaey S, Massaro MA, Chiocca S, Chiesa F, Ansarin M: HPV in oropharyngeal cancer: the basics to know in clinical practice. Acta Otorhinolaryngol Ital 2014, 34:299-309.

7. Yu G, Wang LG, Han Y, He QY: clusterProfiler: an R package for comparing biological themes among gene clusters. OMICS 2012, 16:284-7.

8. Zhang $\mathrm{M}$, Wang J, Gu H: Identification of biological processes and signaling pathways in the stretched nucleus pulposus cells. bioRxiv 2021:2021.11.23.469730.

9. Hanming G: nuotrophils arthritis. Research Square 2021.

10. Palefsky JM: Human papillomavirus-related disease in men: not just a women's issue. J Adolesc Health 2010, 46:S12-9.

11. Aggarwal S, John S, Sapra L, Sharma SC, Das SN: Targeted disruption of PI3K/Akt/mTOR signaling pathway, via PI3K inhibitors, promotes growth inhibitory effects in oral cancer cells. Cancer Chemother Pharmacol 2019, 83:451-61.

12. Jhou AJ, Chang HC, Hung CC, Lin HC, Lee YC, Liu WT, Han KF, Lai YW, Lin MY, Lee CH:

Chlorpromazine, an antipsychotic agent, induces $\mathrm{G} 2 / \mathrm{M}$ phase arrest and apoptosis via regulation of the PI3K/AKT/mTOR-mediated autophagy pathways in human oral cancer. Biochem Pharmacol 2021, 184:114403.

13. Marquard FE, Jucker M: PI3K/AKT/mTOR signaling as a molecular target in head and neck cancer. Biochem Pharmacol 2020, 172:113729.

14. Walker C, Mojares E, Del Rio Hernandez A: Role of Extracellular Matrix in Development and Cancer Progression. Int J Mol Sci 2018, 19.

15. Vilen ST, Salo T, Sorsa T, Nyberg P: Fluctuating roles of matrix metalloproteinase-9 in oral squamous cell carcinoma. ScientificWorldJournal 2013, 2013:920595.

16. Sundquist E, Renko O, Salo S, Magga J, Cervigne NK, Nyberg P, Risteli J, Sormunen R, Vuolteenaho O, Zandonadi F, Paes Leme AF, Coletta RD, Ruskoaho H, Salo T: Neoplastic extracellular matrix environment promotes cancer invasion in vitro. Exp Cell Res 2016, 344:229-40.

17. Morita Y, Hata K, Nakanishi M, Omata T, Morita N, Yura Y, Nishimura R, Yoneda T: Cellular fibronectin 1 promotes VEGF-C expression, lymphangiogenesis and lymph node metastasis associated with human oral squamous cell carcinoma. Clin Exp Metastasis 2015, 32:739-53.

18. Guo C, Liu S, Wang J, Sun MZ, Greenaway FT: ACTB in cancer. Clin Chim Acta 2013, 417:39-44.

19. Oh SY, Kang SM, Kang SH, Lee HJ, Kwon TG, Kim JW, Lee ST, Choi SY, Hong SH: Potential Salivary mRNA Biomarkers for Early Detection of Oral Cancer. J Clin Med 2020, 9.

20. Mao SZ, Fan XF, Xue F, Chen R, Chen XY, Yuan GS, Hu LG, Liu SF, Gong YS: Intermedin modulates hypoxic pulmonary vascular remodeling by inhibiting pulmonary artery smooth muscle cell 
proliferation. Pulm Pharmacol Ther 2014, 27:1-9.

21. Yuan G, Yang S, Gautam M, Luo W, Yang S: Macrophage regulator of G-protein signaling 12 contributes to inflammatory pain hypersensitivity. Ann Transl Med 2021, 9:448.

22. Yuan G, Yang S, Ng A, Fu C, Oursler MJ, Xing L, Yang S: RGS12 Is a Novel Critical NF-kappaB Activator in Inflammatory Arthritis. iScience 2020, 23:101172.

23. Oldham WM, Hamm HE: Heterotrimeric G protein activation by G-protein-coupled receptors. Nat Rev Mol Cell Biol 2008, 9:60-71.

24. Fu C, Yuan G, Yang ST, Zhang D, Yang S: RGS12 Represses Oral Cancer via the Phosphorylation and SUMOylation of PTEN. J Dent Res 2020:22034520972095.

25. Yuan G, Yang S, Yang S: Macrophage RGS12 contributes to osteoarthritis pathogenesis through enhancing the ubiquitination. Genes \& Diseases 2021.

26. Kach J, Sethakorn N, Dulin NO: A finer tuning of G-protein signaling through regulated control of RGS proteins. Am J Physiol Heart Circ Physiol 2012, 303:H19-35.

27. Yuan G, Yang S, Yang S, Ng A, Oursler MJ: RGS12 is a critical proinflammatory factor in the pathogenesis of inflammatory arthritis via acting in Cox2-RGS12-NF kappa B pathway activation loop. J Bone Miner Res: WILEY 111 RIVER ST, HOBOKEN 07030-5774, NJ USA, 2019. pp. 147-.

28. Yuan G, Yang S, Liu M, Yang S: RGS12 is required for the maintenance of mitochondrial function during skeletal development. Cell Discov 2020, 6:59.

29. O'Brien JB, Wilkinson JC, Roman DL: Regulator of G-protein signaling (RGS) proteins as drug targets: Progress and future potentials. J Biol Chem 2019, 294:18571-85.

30. Yuan G, Fu C, Yang ST, Yuh DY, Hajishengallis G, Yang S: RGS12 Drives Macrophage Activation and Osteoclastogenesis in Periodontitis. J Dent Res 2021:220345211045303.

31. Yuan G, Huang Y, Yang ST, Ng A, Yang S: RGS12 inhibits the progression and metastasis of multiple myeloma by driving M1 macrophage polarization and activation in the bone marrow microenvironment. Cancer Commun (Lond) 2022, 42:60-4.

32. Luo R, Jeong SJ, Jin Z, Strokes N, Li S, Piao X: G protein-coupled receptor 56 and collagen III, a receptor-ligand pair, regulates cortical development and lamination. Proc Natl Acad Sci U S A 2011, 108:12925-30.

33. Chen HC, Tseng YK, Shu CW, Weng TJ, Liou HH, Yen LM, Hsieh IC, Wang CC, Wu PC, Shiue YL, Fu TY, Tsai KW, Ger LP, Liu PF: Differential clinical significance of COL5A1 and COL5A2 in tongue squamous cell carcinoma. J Oral Pathol Med 2019, 48:468-76.

34. Lu XZ, Chen WT, Zhang CP: [Investigation of mRNA expression of collagen genes in oral squamous cell carcinoma and paired normal tissue]. Nan Fang Yi Ke Da Xue Xue Bao 2011, 31:1197-9.

35. Hsu LS, Huang RH, Lai HW, Hsu HT, Sung WW, Hsieh MJ, Wu CY, Lin YM, Chen MK, Lo YS, Chen CJ: KLF6 inhibited oral cancer migration and invasion via downregulation of mesenchymal markers and inhibition of MMP-9 activities. Int J Med Sci 2017, 14:530-5. 
36. Grauzam S, Brock AM, Holmes CO, Tiedeken JA, Boniface SG, Pierson BN, Patterson DG, Coaxum SD, Neskey DM, Rosenzweig SA: NEDD9 stimulated MMP9 secretion is required for invadopodia formation in oral squamous cell carcinoma. Oncotarget 2018, 9:25503-16.

37. Chaix A, Zarrinpar A, Panda S: The circadian coordination of cell biology. J Cell Biol 2016, 215:1525.

38. Yuan G, Xu L, Cai T, Hua B, Sun N, Yan Z, Lu C, Qian R: Clock mutant promotes osteoarthritis by inhibiting the acetylation of NFkappaB. Osteoarthritis Cartilage 2019, 27:922-31.

39. Zhu Z, Xu L, Cai T, Yuan G, Sun N, Lu C, Qian R: Clock represses preadipocytes adipogenesis via GILZ. J Cell Physiol 2018, 233:6028-40.

40. Xu L, Cheng Q, Hua B, Cai T, Lin J, Yuan G, Yan Z, Li X, Sun N, Lu C, Qian R: Circadian gene Clock regulates mitochondrial morphology and functions by posttranscriptional way. bioRxiv 2018:365452.

41. Yuan G, Hua B, Yang Y, Xu L, Cai T, Sun N, Yan Z, Lu C, Qian R: The Circadian Gene Clock Regulates Bone Formation Via PDIA3. J Bone Miner Res 2017, 32:861-71.

42. Fan XF, Wang XR, Yuan GS, Wu DH, Hu LG, Xue F, Gong YS: [Effect of safflower injection on endoplasmic reticulum stress-induced apoptosts in rats with hypoxic pulmonary hypertension]. Zhongguo Ying Yong Sheng Li Xue Za Zhi 2012, 28:561-7.

43. Zhu Z, Hua B, Shang Z, Yuan G, Xu L, Li E, Li X, Sun N, Yan Z, Qian R, Lu C: Altered Clock and Lipid Metabolism-Related Genes in Atherosclerotic Mice Kept with Abnormal Lighting Condition. Biomed Res Int 2016, 2016:5438589.

44. Marcheva B, Ramsey KM, Peek CB, Affinati A, Maury E, Bass J: Circadian clocks and metabolism. Handb Exp Pharmacol 2013:127-55.

45. Yuan G, Hua B, Cai T, Xu L, Li E, Huang Y, Sun N, Yan Z, Lu C, Qian R: Clock mediates liver senescence by controlling ER stress. Aging 2017, 9:2647-65.

46. Cai T, Hua B, Luo D, Xu L, Cheng Q, Yuan G, Yan Z, Sun N, Hua L, Lu C: The circadian protein CLOCK regulates cell metabolism via the mitochondrial carrier SLC25A10. Biochim Biophys Acta Mol Cell Res 2019, 1866:1310-21.

47. Zhu Z, Hua B, Xu L, Yuan G, Li E, Li X, Sun N, Yan Z, Lu C, Qian R: CLOCK promotes 3T3-L1 cell proliferation via Wnt signaling. IUBMB Life 2016, 68:557-68.

48. Xie Y, Tang Q, Chen G, Xie M, Yu S, Zhao J, Chen L: New Insights Into the Circadian Rhythm and Its Related Diseases. Front Physiol 2019, 10:682.

49. Wang J, Li S, Li X, Li B, Li Y, Xia K, Yang Y, Aman S, Wang M, Wu H: Circadian protein BMAL1 promotes breast cancer cell invasion and metastasis by up-regulating matrix metalloproteinase 9 expression. Cancer Cell Int 2019, 19:182.

50. Liu Y, Li W, Li X, Tai Y, Lu Q, Yang N, Jiang J: Expression and significance of biglycan in endometrial cancer. Arch Gynecol Obstet 2014, 289:649-55.

\section{Tables 1-2}


Tables 1-2 are available in the Supplementary Files section.

\section{Figures}

Figure 1

Heatmap and volcano plot were created in oral cancer between HPV-negative and HPV-positive patients

(A) Heatmap of significant DEGs. Significant DEGs $(P<0.01)$ were used to construct the heatmap.

(B) Volcano plot for DEGs in oral cancer between HPV-negative and HPV-positive patients. The most significantly changed genes are highlighted by grey dots.

Figure 2

KEGG and GO analyses of DEGs in oral cancer between HPV-negative and HPV-positive patients (A) KEGG analysis, (B) Biological processes, (C) Cellular components, (D) Molecular functions.

\section{Figure 3}

The PPI network analyses of DEGs in oral cancer between HPV-negative and HPV-positive patients The cluster (A) and cluster (B) were constructed by MCODE.

Figure 4

Reactome map representation of the significant biological processes in oral cancer between HPVnegative and HPV-positive patients

\section{Supplementary Files}

This is a list of supplementary files associated with this preprint. Click to download.

- SupplementalTableS1.csv

- Onlinefloatimage5.png 
- Onlinefloatimage6.png

Page 10/10 